\title{
'La chiamiamo con entrambi i nomi': eponimia ed eteronimia tra Nemesis e Adrasteia
}

'She is Called by a Double Name'. Eponymy and Heteronymy between Nemesis and Adrasteia

\section{Daniela Bonanno}

\section{CpenEdition}

\section{Journals}

\section{Edizione digitale}

URL: https://journals.openedition.org/mythos/2753

DOI: $10.4000 /$ mythos.2753

ISSN: 2037-7746

\section{Editore}

Salvatore Sciascia Editore

Notizia bibliografica digitale

Daniela Bonanno, «'La chiamiamo con entrambi i nomi': eponimia ed eteronimia tra Nemesis e Adrasteia», Mythos [Online], 14 | 2020, online dal 31 décembre 2020, consultato il 07 octobre 2021. URL: http://journals.openedition.org/mythos/2753 ; DOI: https://doi.org/10.4000/mythos.2753

Questo documento è stato generato automaticamente il 7 octobre 2021.

Mythos 


\section{'La chiamiamo con entrambi i nomi': eponimia ed eteronimia tra Nemesis e Adrasteia}

'She is Called by a Double Name'. Eponymy and Heteronymy between Nemesis and Adrasteia

Daniela Bonanno

\section{Introduzione ${ }^{1}$}

1 Esaminare e decodificare le strategie sottese alla denominazione del divino nel mondo greco - si sa - è operazione complessa e lo è tanto più quando a doversi orientare nella moltitudine dei diversi attributi onomastici, assegnati a una potenza ${ }^{2}$, non sempre risulti chiaro nelle fonti il rapporto tra il teonimo e il suo appellativo o quando l'uno sia impiegato in luogo dell'altro ${ }^{3}$. È stato sottolineato di recente, come il tradizionale binomio teonimo+epiteto/epiclesi sia del tutto inadeguato a rendere conto dell'immensa varietà che governa le logiche di denominazione, evocazione $\mathrm{e}$ qualificazione di una potenza divina ${ }^{4}$. Accostare al nome di una divinità un aggettivo $o$ un sostantivo o il nome di un altro dio non è mai un atto banale o scontato; significa celebrare episodi della sua vicenda mitica; declinarne le competenze anche in rapporto ad altri dèi; definire le prassi rituali e cultuali che lo riguardano e la capacità di presa su un territorio, sulla vita degli uomini o su una singola fase della loro esistenza; significa, in ultima analisi, dare voce alle istanze di comunicazione dei devoti, nella loro relazione con il divino ${ }^{5}$.

2 Fra le potenze greche, Nemesis non è certo fra quelle che presentano un corredo troppo ampio di attributi onomastici atti a indicarne le funzioni, le vicende mitiche oppure ad ancorarne la potenza a questa o a quell'area geografica. Tuttavia, benché piuttosto limitato sia, in questo caso, il ventaglio delle possibili denominazioni, una su tutte sembra prevalere, offrendoci materiale per l'analisi di un caso studio senz'altro efficace ai fini dell'esplorazione della rete di relazioni che intercorrono tra un teonimo e le sue 
possibili designazioni. Infatti, tra gli appellativi impiegati per evocare Nemesis, un ruolo di primo piano, nelle fonti, come nella storia degli studi ${ }^{6}$, è senz'altro riconosciuto ad Adrasteia, che risulta impiegato ora come eteronimo di Nemesis, ora come eponimo, ora infine come teonimo indipendente ${ }^{7}$.

In un passo delle Res gestae, Ammiano Marcellino, greco della città di Antiochia in Siria e ufficiale dell'esercito romano imperiale, narrando dell'esecuzione di Gallo, nipote dell'imperatore Costantino e fratellastro di Giuliano, commenta la terribile sorte toccata ai suoi due assassini e individua nell'azione di Adrasteia le atroci sofferenze che li portarono alla mortes:

Haec et huius modi quaedam innumerabilia ultrix facinorum impiorum bonorumque praemiatrix, aliquotiens operatur Adrastia (atque utinam semper): quam vocabulo duplici etiam Nemesim appellamus: ius quoddam sublime numinis efficacis, humanarum mentium opinione lunari circulo superpositum, vel ut definiunt alii, substantialis tutela generali potentia partilibus praesidens fatis, quam theologi veteres fingentes Iustitiae filiam ex abdita quadam aeternitate tradunt omnia despectare terrena. Haec ut regina causarum, et arbitra rerum ac disceptatrix, urnam sortium temperat, accidentium vices alternans, voluntatum nostrarum exorsa interdum alio quam quo contendebant exitu terminans, multiplices actus permutando convolvit. Eademque necessitatis insolubili retinaculo mortilitatis vinciens fastus, tumentes in cassum, et incrementorum detrimentorumque momenta versabilis librans (ut novit), nunc erectas eminentium cervices oppromit et enervat, nunc bonos ab imo suscitans ad bene vivendum extollit.

Queste ed altre innumerevoli opere del genere compie alle volte (e magari le compisse sempre) Adrastia, che punisce gli atti empi e premia quelli buoni. Noi la chiamiamo con duplice nome anche Nemesis. Essa è una legge sublime della divinità onnipotente ed ha la sua sede, a quanto si ritiene, al di sopra dell'orbita lunare. Altri invece la definiscono un'ipostasi che, dotata di potere universale, difende i destini dei singoli. I teologi antichi l'immaginarono figlia della Giustizia e narrano che dalle epoche più remote osserva dall'alto le vicende terrene. Costei, in quanto regina delle cause e arbitra delle vicende umane, agita l'urna delle sorti e provoca $\mathrm{i}$ mutamenti della fortuna e, portando alle volte le iniziative della nostra volontà ad un termine diverso da quello propostoci, trasforma e sconvolge molte azioni. Essa pure lega l'orgoglio dei mortali, che invano insuperbisce, con l'indissolubile freno del destino e, pesando mutevole sulla bilancia abbatte e piega le cervici superbe dei potenti, ora solleva i buoni dal fondo e li conduce ad una vita felice.

Amm. Rerum Gestarum Libri, XIV 11, 25-26 (trad. A. Selem-Utet)

Le considerazioni di Ammiano, in merito alle prerogative di questa forza composita che presiede all'alternarsi delle sorti sanzionando i superbi, e il riferimento al duplice nome con cui la si designa lasciano trasparire le tracce di un dibattito, sul rapporto tra le due potenze, che deve avere attraversato l'Antichità'. Nella rappresentazione dello storico, in cui evidenti sono gli echi all'immagine di Nemesis, quale emerge dagli Inni orfici o dall'inno dedicatole dal poeta di età adrianea Mesomede, chiara appare la sovrapposizione di questa divinità ad Adrasteia, l'una essendo ormai assunta quale eteronimo dell'altra ${ }^{10}$.

Il confronto con le testimonianze di epoca precedente restituisce però un quadro ben più articolato, mostrando come le due potenze non siano state sempre considerate intercambiabili, ma come esse fossero celebrate e invocate separatamente. Ricostruire la storia di questa trasformazione consente di mettere in evidenza i molteplici, e talora imprevedibili, esiti, cui le forme di denominazione del divino possono andare incontro, 
lasciando emergere connessioni nascoste tra le loro differenti declinazioni. Si procederà quindi con l'esame delle testimonianze, nel tentativo di individuare le tappe di una trasformazione che ha contribuito a modificare il profilo stesso di una potenza, la sua percezione da parte dei devoti e la capacità che le era riconosciuta di incidere sulle vicende umane.

\section{Adrasteia, Nemesis e il controllo della parola}

6 Il nome Adrasteia ricorre indipendentemente da quello di Nemesis già nel Prometeo incatenato attribuito a Eschilo. Nel dramma, il Coro, quasi impotente, tenta di ricondurre alla ragione il titano Prometeo che, recalcitrante rispetto al dominio universale di Zeus, preconizza la prossima fine del suo regno:

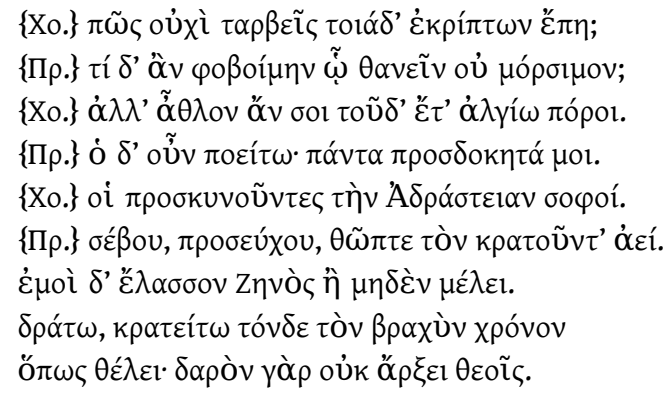

7 In questi versi, l'eloquio arrogante di Prometeo sollecita il coro delle Oceanine a evocare il nome di Adrasteia. Da questo scambio serrato emergono i tratti peculiari di una potenza, preposta al controllo della parola e di fronte alla quale conviene piegarsi

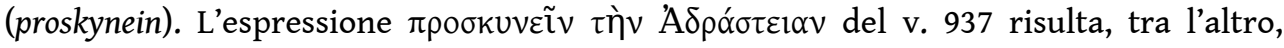
frequentemente impiegata nelle fonti, come formula apotropaica, pronunciata per stornare la punizione divina da chi consapevolmente si lascia andare ad autoelogi e panegirici verbali ${ }^{11}$. Questa forza, rappresentativa del dominio indiscusso e inevitabile del Cronide $^{12}$, compare anche nel Reso pseudoeuripideo, un dramma, la cui trama sembra ruotare proprio su un uso imprudente della parola: la protervia verbale del tracio Reso, soccorritore tardivo dei Troiani, convinto che sarebbe riuscito a respingere i Greci dalle mura di Troia in un sol giorno, si scioglie nella morte ingloriosa cui lo condannano nella notte gli eroi greci Odisseo e Diomede, entrati furtivamente nel suo accampamento. La colpa di questo indegno omicidio ricade sul troiano Ettore, che all'alleato aveva rinfacciato la sua intempestività. Storia di tradimenti e di traditori, di inganni e travestimenti, di promesse fallaci, di accuse false e infamanti, il Reso si conclude con l'intervento della Musa, madre dell'eroe tracio, entità tradizionalmente legata a un uso veridico e autentico della parola di ispirazione divina ${ }^{13}$. In quest'opera il ricorrere del nome di Adrasteia non sembra irrilevante: invocata, questa volta, come 
figlia di Zeus, essa è precisamente designata come una divinità che interviene sulla parola arrogante ${ }^{14}$.

8 A livello cultuale, Adrasteia è menzionata, in un'iscrizione della seconda metà del $\mathrm{V}$ sec., insieme ad altre divinità, dai cui santuari gli Ateniesi presero in prestito del denaro per finanziare le prime fasi della guerra del Peloponneso. Il suo nome è annoverato tra gli alloi theoi, insieme alla tracia Bendis, che era onorata presso il Pireo ${ }^{15}$. Come le altre potenze ricordate in questo documento, anche Adrasteia doveva avere un luogo di culto, che ricadeva nell'amministrazione della città, distinto da quello dedicato alla Nemesis di Ramnunte, sulla costa nord orientale dell'Attica ${ }^{16}$.

Di certo, però, la menzione più celebre di Adrasteia si trova nel Fedro di Platone all'interno di una lunga riflessione sulla natura immortale dell'anima. Nel dialogo platonico, alla volontà della dea è attribuito il thesmos che regola la metempsicosi delle anime. Si tratta di un passo che, per i contenuti che veicola, è stato fondamentalmente esaminato nel quadro delle testimonianze "orfiche" ${ }^{17}$. Tuttavia, un allargamento dell'analisi al contesto argomentativo in cui il brano risulta inserito potrà forse favorire il reperimento di altre chiavi di lettura.

Ebbene, in un dialogo come il Fedro platonico, dedicato ai rischi e agli infingimenti della retorica, ma anche alla rivalutazione di essa come psychagogia, ovvero «l'arte di condurre le anime» ${ }^{18}$, Adrasteia è chiamata in causa - forse non a caso - per la capacità che ha di determinare la permanenza delle anime nei corpi degli uomini, a seconda del grado di visione divina cui esse abbiano partecipato, prima di impiantarsi in essi. Per il tramite di Socrate, Platone spiega, infatti, che l'anima dei mortali assomiglia a un carro alato, lanciato all'inseguimento del corteo divino. Questo carro, guidato da un auriga, è trascinato da una coppia di cavalli, di cui uno di razza eccellente e l'altro di cattiva razza. Quest'ultimo trascina il carro verso il basso, allontanando così l'anima dalla visione divina, in una caduta vertiginosa che la porterà a perdere ali $^{19}$.

11 Ora, l'anima che, nel suo percorso sarà riuscita, nonostante le tensioni contrarie, a tenere il passo al seguito del corteo divino e a contemplare una parte consistente della verità, si impianterà nel corpo di colui che è destinato ad essere un filosofo e un amante del bello; le anime, invece, che, per accidenti vari, abbiano partecipato di una visione ridotta della verità e del bello divino si impianteranno in esseri via via più modesti dal re, al legislatore, al politico, all'atleta, fino ad arrivare alle più infime categorie rappresentate dal sofista e dall'adulatore del popolo per finire con il tiranno ${ }^{20}$. Nella prospettiva del Fedro, incentrata com'è sull'opposizione tra una retorica unicamente finalizzata all'efficacia tecnica del discorso, senza alcun rapporto con la verità e una retorica psicagogica diretta a influenzare e condurre le anime, l'evocazione di una potenza come Adrasteia preposta al controllo delle parole, è assolutamente significativa. La sua azione si concretizza precisamente nell'organizzazione di una gerarchia di anime, alla cui sommità è collocato il filosofo, cui si attribuisce una memoria piena del bello e del vero e i cui discorsi non possono che conformarsi alla verità; mentre al gradino più basso, si trovano le anime del sofista, dell'adulatore del popolo o del tiranno, figure caratterizzate, tra l'altro, per il loro uso strumentale e distorto della parola ${ }^{21}$. Si riconosce in questi termini ad Adrasteia la capacità di guardare nell'animo umano e di identificare la parte divina che essa contiene, ma soprattutto di comprendere a che punto le parole pronunciate corrispondano alle verità assimilate al seguito del corteo divino. 
12 In termini simili, del resto, anche Socrate dichiara nella Repubblica di Platone di

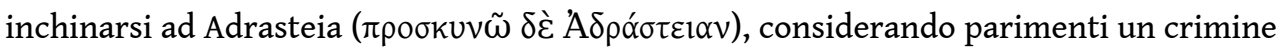
quello di uccidere un uomo e di ingannarlo su quanto è buono, bello e legalmente giusto $^{22}$. Si ritrovano, ancora una volta, nella riflessione platonica, le prerogative di una divinità che riconosce l'autenticità degli individui, che ascolta, classifica e soprattutto declassa. La formulazione stessa del suo nome allinea automaticamente la realtà ai fatti; da qui ha origine la pulsione istintiva ad abbassarsi, a ridimensionarsi rispetto alle parole pronunciate.

Questo controllo sull'arroganza verbalmente espressa costituisce precisamente il trait d'union tra Nemesis e Adrasteia. Benché in maniera diversa, Nemesis ricopre una competenza analoga. È sufficiente a questo proposito fare riferimento ai versi delle Fenicie di Euripide e, in particolare, alle preghiere di Antigone, allorché osserva impotente l'assedio di Tebe, minacciata dagli eroi venuti da Argo, guidati da Polinice, che attaccano ciascuna delle sue sette porte.

Alla vista di Capaneo, che con il suo sguardo misura le mura delle città, Antigone dalla sommità del palazzo invoca Nemesis:

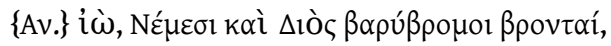

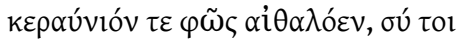

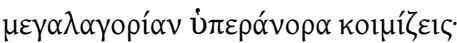

Nemesis e tuoni altisonanti di Zeus

luce fiammeggiante della folgore, tu che

metti a tacere il discorso superbo violento.

Eur. Phoen. 182-185 (trad. personale)

In questo passo, Euripide attribuisce a Nemesis e al fulmine di Zeus la funzione di ridurre al silenzio la megalagoria, il discorso violento e superbo. Tale sfera d'azione è analogamente riconosciuta a Nemesis in altre fonti ${ }^{23}$, per esempio proprio nelle Leggi di Platone, in cui la dea, designata come "messaggera di Dike", è descritta come colei che

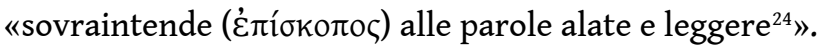

\section{Nemesis in azione nelle Storie di Erodoto}

Un passo del primo libro delle Storie di Erodoto ci offre una testimonianza fondamentale per indagare la maniera in cui agisce nemesis, in quanto forza, consegnandoci così una griglia interpretativa essenziale per la comprensione della sua efficacia nel pensiero religioso dei Greci. Il contesto è quello del dialogo celeberrimo tra il legislatore ateniese Solone e il sovrano lidio Creso, inserito nel primo libro dell'opera erodotea $^{25}$. Creso domanda a Solone, se nei suoi viaggi avesse mai «visto un uomo più

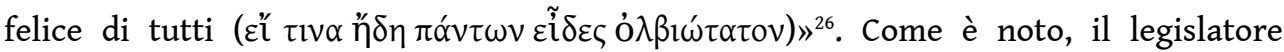
risponde, riportando due esempi di felicità rappresentati da uomini comuni ${ }^{27}$. Tale risposta suscita immediatamente l'irritazione di Creso che vede sottostimata la sua ricchezza e le sue fortune, ritenute addirittura inferiori a quelle di privati cittadini. Solone spiega allora al sovrano lidio qual è la vera natura del divino, che definisce phthoneron e tarachodes («invidiosa e capace di stravolgere»), e conclude precisando che è necessario attendere il termine della vita di un uomo per stabilire se è stato veramente felice ${ }^{28}$. 

maggiore frequenza. Esso indica il possesso in grande quantità di un bene; una condizione che si iscrive con chiarezza nel registro semantico dell'abbondanza ${ }^{31}$. Il sostantivo phthonos indica, per converso, la mancanza di qualcosa che bisogna compensare. Questa condizione descritta per marcare un modo di essere, un'attitudine degli dèi, è lucidamente analizzata nel discorso tenuto da un altro grande saggio dell'opera erodotea, il persiano Artabano, zio paterno di Serse. Egli si rivolge al nipote Serse, in procinto di organizzare una spedizione contro la Grecia e tenta di dissuaderlo, o quanto meno di convincerlo a temporeggiare, spiegandogli le conseguenze che derivano dall'aspirazione a un potere così grande ${ }^{32}$. È esattamente tramite il ricorso al termine phthonos, che egli spiega al sovrano persiano l'attitudine degli dèi a colpire con fulmini gli animali che si distinguono tra gli altri, o gli edifici e gli alberi che si elevano troppo verso le altezze celesti. In questo modo, lo phthonos divino può fare cadere nel panico un grande esercito, anche con un tuono, portandolo a una misera disfatta. E aggiunge: «[...] il dio non permette che altri, al di fuori di lui, nutrano pensieri di

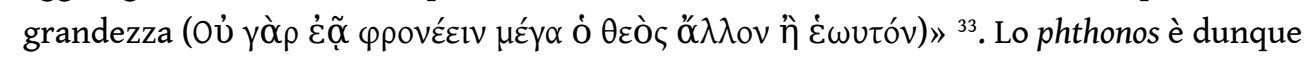
il segno di uno splendore divino che si riduce a causa del mega phroneein degli uomini, a sua volta incoraggiato da una prosperità prolungata e, di conseguenza, dall'oblio dei limiti che caratterizzano la condizione umana ${ }^{34}$.

19 Analogamente, all'origine delle disgrazie di Creso vi è per l'appunto il suo mega phroneein, assolutamente mal riposto, tanto più che sulla sua testa pesa un regime molto più complesso di causalità ${ }^{35}$, che lo porterà dritto verso la catastrofe. Come saprà successivamente, sul destino della sua monarchia pesa l'errore ancestrale compiuto dal suo antenato Gige, colpevole di avere usurpato una time regale che non gli apparteneva ${ }^{36}$.

Quando incontra Solone, Creso non è ancora cosciente di quello che lo aspetta, anzi contrariato dalle risposte dell'Ateniese, lo congeda bruscamente, ma aggiunge Erodoto:

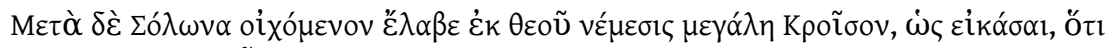

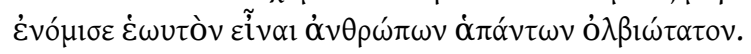

Dopo la partenza di Solone la grande nemesis del dio colse Creso, come sembra, poiché aveva ritenuto di essere il più felice di tutti gli uomini.

Hdt. I 34, 1 (trad. personale)

Il sostantivo nemesis è un hapax nelle Storie e il suo ricorrere, proprio all'interno di una sezione dell'opera cui è riconosciuta un'assoluta rilevanza per la comprensione del programma storiografico di Erodoto, sollecita a indagarne gli esiti ${ }^{37}$. Il termine definisce la sanzione, annunciata per il tramite di un sogno che rivela al sovrano lidio l'aletheia sulle disgrazie che si sarebbero abbattute su di lui, causando la perdita del figlio, ucciso accidentalmente dal suo ospite, il supplice di origine frigia Adrasto ${ }^{38}$. 
La più antica attestazione in cui Nemesis e Adrasteia si trovano l'una accanto all'altra o meglio l'una come epiclesi dell'altra - si trova in un frammento di Antimaco di Colofone, citato per la prima volta da Strabone, ma noto anche per altre vie ${ }^{42}$ :

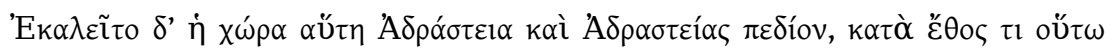

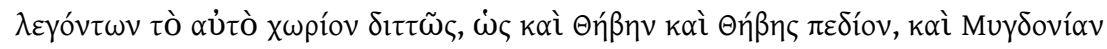

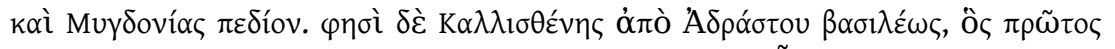

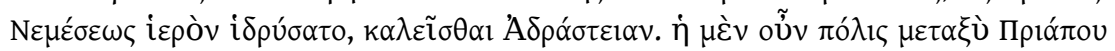

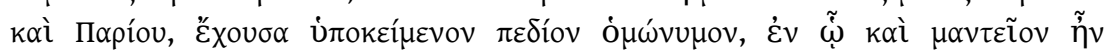

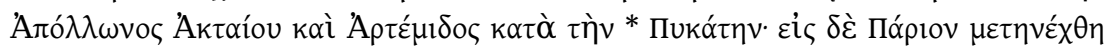

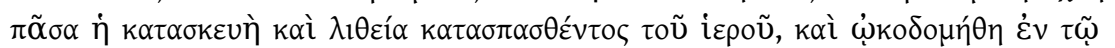

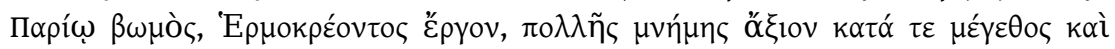

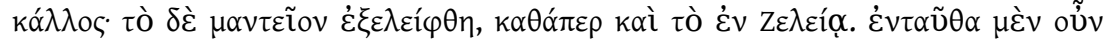

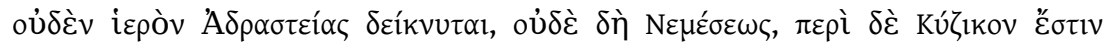

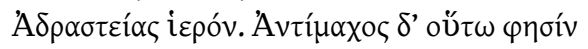

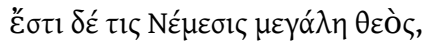

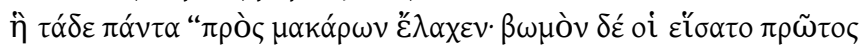

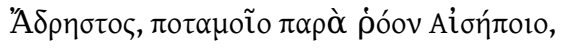

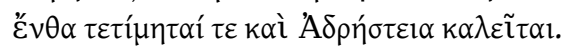


Questa regione è chiamata Adrasteia e piana di Adrasteia, secondo l'uso di chiamare lo stesso luogo in due modi: come Tebe e la piana di Tebe, Migdonia e la piana Migdonia. Callistene afferma che Adrasteia prese nome dal re Adrasto, che per primo elevò un santuario di Nemesis. La città stessa di Adrasteia, tra Priapo e Pario, è situata nella piana omonima, in cui, proprio di fronte, Pattia si trovava un oracolo di Apollo Acteo e di Artemide*. Essendo stato distrutto completamente il santuario, tutto l'allestimento e le pietre furono trasportate a Pario per costruirvi un altare, opera di Ermocreonte, degno di restare a lungo nella memoria per la sua bellezza e per le sue dimensioni. Quanto all'oracolo con il tempo è stato abbandonato come quello a Zeleia. Qui non vi è più nessun santuario né di Adrasteia né di Nemesis, invece nei pressi di Cizico vi è un santuario di Adrasteia. Antimaco dice così:

"Nemesis è una grande dea,

che ha ricevuto tutti i suoi doni dagli immortali:

Adrasto per primo le dedicò un altare presso la corrente del fiume Esepo, qui è onorata e invocata anche come Adrasteia".

Strab. XIII 1, 13 (trad. personale)

La ricostruzione del geografo è problematica per tutta una serie di ragioni: intanto, una corruttela nel testo rende più incerta la ricostruzione del suo ragionamento, ma soprattutto appare difficile individuare con precisione l'area della Troade in cui si sorgeva il santuario di Nemesis. Inizialmente, Strabone sembra collocarlo, sulla base della citazione di Callistene, nella città di Adrasteia, sita tra Priapo e Pario, e ne attribuisce l'iniziativa al re Adrasto che, per primo, elevò uno hieron a Nemesis nella stessa regione in cui si trovava un manteion di Apollo Acteo e di Artemide; in un secondo momento, però il Geografo precisa che, distrutto il santuario l'allestimento e le pietre furono trasportate a Pario nella parte occidentale della Troade dove venne costruito un altare degno di menzione, e aggiunge che nella regione non c'era più nessun santuario né di Adrasteia né di Nemesis, ma che uno hieron di Adrasteia si trovava nei pressi di Cizico, quindi proprio in prossimità del monte omonimo ${ }^{43}$. Infine Strabone riporta un frammento del poeta Antimaco di Colofone, vissuto tra V e IV sec. e autore di una Tebaide, ma anche di altre opere, tra cui alcuni studi sui poemi omerici. Nei versi di Antimaco, riportati dal geografo, si ricorda la dedica a Nemesis da parte di Adrasto, colui che per primo eresse, sulle correnti del fiume Esepo, questa volta, nella parte orientale della Troade, un altare, e che la dea vi è onorata lì anche come Adrasteia.

Ora, l'analisi del passo di Strabone sembrerebbe suggerire una presa di posizione da parte dell'autore che, con la citazione del passo di Callistene prima e con quella di Antimaco poi, pare voler informare il lettore dell'esistenza di due, forse tre luoghi, in cui Nemesis era onorata nella regione come Adrasteia; uno spazio fluido che, forse, in ragione di un esasperato ricorso all'omonimia o ai rapporti di forza interni all'area in questione, restava difficilmente identificabile. Inoltre, secondo Strabone e le sue fonti, Nemesis doveva il suo attributo onomastico all'eroe eponimo della regione. L'identità di questo sovrano Adrasto non è precisata, così come ignote sono le ragioni per le quali aveva deciso di fondare il culto. Il Catalogo delle navi, nel secondo libro dell'Iliade, ci fornisce qualche dettaglio utile a questo proposito, ricordando due comandanti troiani, provenienti proprio dalla regione Adrasteia, vicino al fiume Esepo ${ }^{44}$ : Adrasto e Anfio, figli di Merope. Quest'ultimo, esperto nell'arte mantica, conoscendo il destino che attendeva i due giovani, non voleva lasciarli partire in guerra, ma essi non vollero prestargli ascolto: «li spingevano le Chere di nera morte» ${ }^{45}$. La menzione del fiume Esepo, nel frammento di Antimaco, che rinvia direttamente al Catalogo delle navi, dovrebbe indurre a identificare tale Adrasto con l'eroe di cui parla Omero. 
Purtroppo, però, a complicare il quadro interviene la versione del lessicografo Arpocrazione (II sec. a.C.) che cita ancora una volta Antimaco, con l'aggiunta di un'ulteriore precisazione:

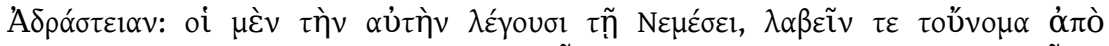

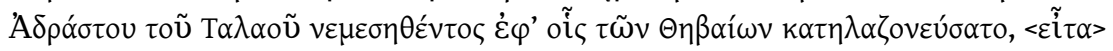

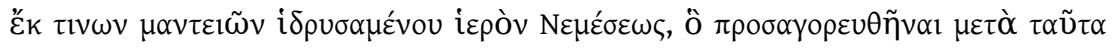

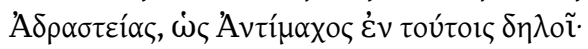

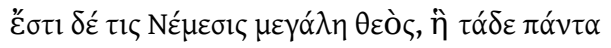

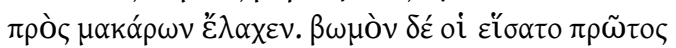

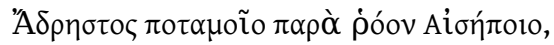

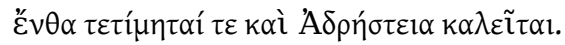

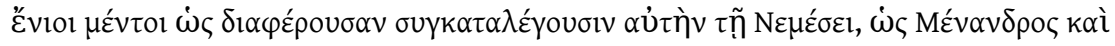

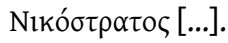

Adrasteia: alcuni dicono che è la stessa di Nemesis e che prende il nome da Adrasto figlio di Talao che era stato fatto oggetto di nemesis, per essersi vantato contro $\mathrm{i}$ Tebani, quindi in seguito ad alcuni oracoli costruì un santuario che poi fu detto di Adrasteia, come dice chiaramente Antimaco in questi versi:

"C'è una grande divinità Nemesis, che ha avuto in sorte tutti questi doni da parte degli immortali. Per primo, le dedicò un altare Adrasto presso la corrente del fiume Esepo.

Qui è onorata e invocata anche come Adrasteia".

Comunque alcuni, come Menandro e Nicostrato, la catalogano come differente da Nemesis [...].

Harp. s.v. Adrasteia (trad. personale)

La voce del lessicografo attribuisce ad Antimaco il dato in base al quale Nemesis avrebbe preso la sua denominazione dall'Adrasto argivo, figlio di Talao, la cui vicenda eroica, brevemente menzionata con l'allusione alla sfortunata impresa dei Sette a Tebe, lo avrebbe indotto, sulla base di alcuni oracoli, a erigere un santuario in onore della dea. In realtà, un'analisi filologica più attenta, condotta da Victor Matthews, ultimo editore di Antimaco ${ }^{46}$, ha mostrato, in primo luogo, in modo del tutto convincente, che il riferimento all'Adrasto argivo, nella voce lessicografica, altro non è che il frutto di una maldestra interpolazione; $\mathrm{e}$ in secondo luogo, che l'Adrasto cui si riferiscono i versi di Antimaco, collegato com'è alla riva del fiume Esepo, può benissimo essere sempre l'omerico figlio di Merope o, tutt'al più, il suo avo, padre di Euridice moglie di Ilo, di cui parla Apollodoro ${ }^{47}$. Ma anche senza volere postulare l'interpolazione del testo di Arpocrazione, in cui il riferimento all'Adrasto argivo compare solo nell'epitome, è un dato di fatto che la relazione tra Nemesis e le tristi vicende del figlio di Talao, cominciano ad apparire nelle rielaborazioni dei lessicografi e dei paremiografi di età imperiale, come Pausania attico, Elio Dionisio, Zenobio e Diogeniano, nonché nella paremiografia tarda di età bizantina ${ }^{48}$. Era questo un fortunato fraintendimento cui si prestava l'etimologia stessa del nome Adrasto, capace di parlare molto chiaramente alle orecchie dei Greci. L'antroponimo coincide con l'aggettivo composto derivato dalla radice dras- del verbo $\delta 1 \delta \rho \alpha ́ \sigma \kappa \omega$ fuggire, preceduto da alfa privativo e seguito dal suffisso -tos, indicante la possibilità. L'aggettivo ricorre nella letteratura greca soltanto un paio di volte, e in particolare nel VI libro delle Storie di Erodoto ${ }^{49}$, in riferimento all'incapacità attribuita dagli Sciti agli Ioni di fuggire, una volta ridotti in schiavitù. L'eroe argivo portava quindi iscritto nel suo nome l'idea di una fuga impossibile da realizzare. È stato già sottolineato da altri come l'etimologia di questo nome non sia del 
tutto trasparente, significando tanto "l'essere incapace" o "non incline alla fuga", quanto "quanto qualcosa da cui non si può sfuggire" ${ }^{50}$.

A dispetto di queste incertezze, la riflessione stoica, che indica con decisione Adrasteia come un'altra maniera di chiamare Zeus, identificando in lui una potenza cui nessuno

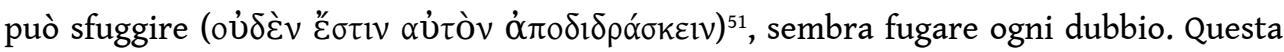
idea di una forza inevitabile da cui è non possibile nascondersi sarà variamente ripresa, anche nelle epoche successive ${ }^{52}$, portando forse prove a sostegno di una solida connessione tra il nome della divinità e le vicende del sovrano argivo, che troviamo poi, a distanza di secoli, pienamente rielaborata e maturata in questo esercizio del retore Libanio, contemporaneo di Ammiano Marcellino e originario della stessa città di Antiochia ${ }^{53}$ :

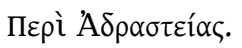

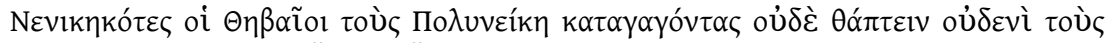

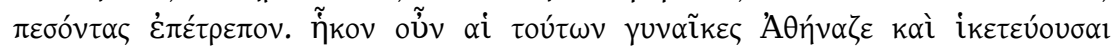

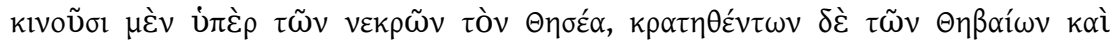

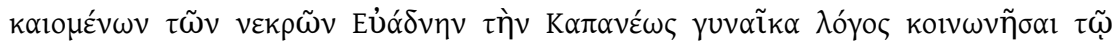

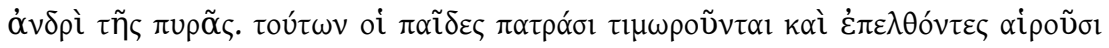

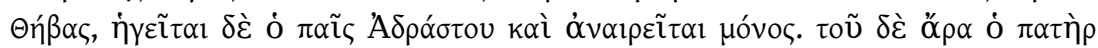

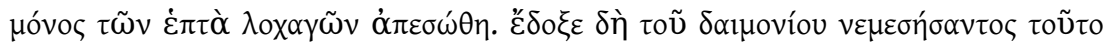

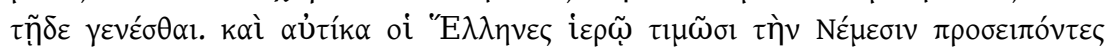

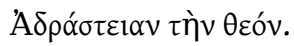

\section{Su Adrasteia}

Dopo avere vinto coloro che cercavano di portare al potere Polinice, i Tebani non consentirono a nessuno di seppellire i caduti. Così le loro vedove giunsero ad Atene e, supplicando Teseo, suscitarono la loro compassione per i defunti, ma quando i Tebani furono sconfitti e i loro corpi vennero bruciati, la storia vuole che Evadne, moglie di Capaneo, condivise la pira funebre con il marito. I figli di questi uomini cercarono vendetta per i loro padri e, dopo averla attaccata, conquistarono Tebe, li guidava il figlio di Adrasto e fu il solo ad essere ucciso. Mentre il padre di costui fu l'unico dei sette a salvarsi. Sembrò che un daimon indignatosi mutasse le cose in tal modo e subito i Greci onorarono con un santuario Nemesis, rivolgendosi alla dea Adrasteia.

Lib. Progymn. 2, 10 (trad. personale)

Il triste destino dell'Adrasto argivo, unico superstite di una disastrosa spedizione contro Tebe, si rifletteva nelle drammatiche conseguenze che portarono alla morte del suo giovane figlio Egialeo, il solo a trovare la morte nel gruppo degli Epigoni, partiti alla volta di Tebe, per vendicare i padri ${ }^{54}$. L'evento che trasformò l'iniziale vittoria dei Tebani sui Sette in una successiva sconfitta da parte della generazione successiva e il destino occorso ad Adrasto e a suo figlio, sembrò ai Greci opera dell'intervento di un «daimon indignato»: una Nemesis che colpisce il responsabile diretto dell'atto, eliminando la sua progenie come già nel caso di Creso. È una Nemesis che trae il suo nome dalla particolare vicenda del celebre Adrasto della saga tebana, divinità eletta, in epoca più tarda, a paradigma di una sanzione divina ineluttabile in cui etimologia e racconto mitico finiscono per formare un riuscito binomio didascalico utile a illustrare le modalità di azione di una precisa potenza divina, rispondendo così agli interrogativi che i Greci si ponevano in merito al rapporto tra responsabilità umana e causalità divina $^{55}$. 


\section{Conclusioni}

Se quindi ancora nel corso del $\mathrm{V}$ secolo, Nemesis e Adrasteia sono evocate separatamente l'una dall'altra, come divinità indipendenti, già nel corso del IV secolo, $\mathrm{i}$ loro nomi cominciano a comparire affiancati, nelle testimonianze letterarie ed epigrafiche, forse in ragione dei loro campi d'azione liminali e talora sovrapponibili o con lo scopo di amplificare la portata dell'invocazione, intercettando la potenza dell'una e dell'altra.

Un paio di iscrizioni provenienti dall'isola di Cos consentono di ampliare la riflessione in merito: si tratta di due testi, databili l'uno alla seconda metà del II sec. a.C. e l'altro alla prima metà del I sec. a.C. ${ }^{56}$ In entrambi i casi si menzionano sacrifici di un valore preciso da dovere dedicare da parte di diverse categorie di individui: nella prima iscrizione (IG XII 4,318), si indicano alcune persone designate da banchieri e coloro che possiedono una banca, ma anche degli schiavi affrancati. Nella seconda iscrizione, sul lato anteriore di una stele di marmo opistografa (IG XII 4, 325), il sacrificio dei banchieri è preceduto da quello di impresari che hanno ottenuto in appalto un cantiere pubblico $^{57}$. La verifica dell'avvenuto adempimento dell'offerta sacrificale da parte degli affrancati e degli impresari è affidata, nelle due iscrizioni, rispettivamente ai tesorieri e agli architetti che non possono procedere alla registrazione dell'atto, prima che il sacerdote lo abbia dichiarato pubblicamente e reso manifesto: è stabilita come pena una sanzione salatissima da pagare ad Adrasteia e a Nemesis. Non è chiaro chi siano in questi casi i soggetti da multare, se gli individui tenuti a compiere il sacrificio o coloro che ne hanno registrato l'atto, prima di una corretta denuncia verbale da parte dell'autorità designata. Nell'uno e nell'altro caso, tuttavia, le due divinità, evocate in un unico binomio onomastico, sembrano chiamate a intervenire laddove vi sia il rischio dell'illegittima attribuzione di una time, sanzionando, inesorabilmente, un'inefficace articolazione tra processo verbale e registrazione di atti pubblici; tra parole e realtà dei fatti ${ }^{58}$.

Non stupisce quindi, alla luce di queste considerazioni, come la prossimità tra Nemesis e Adrasteia possa essersi trasformata nel corso del tempo e, a seconda dei contesti, in una vera e propria sovrapposizione. L'una viene evocata insieme all'altra o diventa l'eteronimo dell'altra, inglobando, nel riferimento all'Adrasto argivo, il paradigma di una sanzione divina ineluttabile, in cui il significato etimologico si intreccia al racconto mitico per costruire un nesso didattico molto più complesso di quanto all'inizio la modesta storia dell'omerico figlio di Merope potesse suggerire: un esempio efficace per dirla con J. Scheid e J. Svenbro - della capacità generativa del mito che i nomi greci possiedono ${ }^{59}$.

Le stesse pratiche rituali, connesse ai nomi delle due divinità che prevedevano l'uso del corpo - quella cioè di prosternarsi ad Adrasteia e quella che le fonti evocano spesso in relazione a Nemesis e che marca gran parte delle sue rappresentazioni iconografiche,

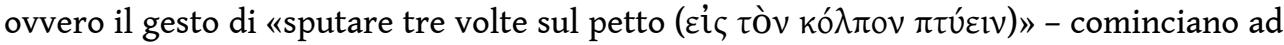
essere attribuite tanto all'una quanto all'altra, finendo, in qualche modo, per chiarirsi reciprocamente ${ }^{60}$. Infatti, la stessa disponibilità dichiarata verbalmente ad inchinarsi, e ad abbassarsi di fronte a una potenza percepita come ferma sanzionatrice da parte di coloro, che si elevano al di sopra di quanto consentito ai mortali, sembra corrispondere, nelle finalità, al gesto che si compie in omaggio a Nemesis. L'atto sembra, infatti, coincidere con una forma aumentata di proskynesis, una deminutio capitis che comporta 
un'ulteriore pratica di degradazione al cospetto del divino, che consisteva per l'appunto nello sputarsi addosso tre volte, in segno di ostilità e di disprezzo verso se

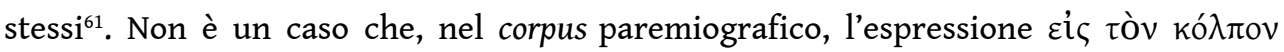
$\pi \tau u ́ \varepsilon \imath v$ finisca per essere illustrata dalla formula: oủ $\mu \varepsilon \gamma \alpha \lambda$ $\rho \dot{\rho} \eta \mu o v \varepsilon \tilde{v} v$, «non vantarsi $\rangle^{62}$.

Al termine di questo percorso - riconosco - punteggiato di ombre e di incertezze, quello che di certo emerge con chiarezza è che le diverse maniere di nominare il divino sono il risultato di un processo complesso e imprevedibile, la cui interpretazione, tanto quella degli antichi quanto quella dei moderni, è costellata di accidenti e condizionata da molteplici variabili: i cambiamenti storici, l'affermarsi di certe tradizioni a scapito di altre, l'influenza delle élites intellettuali.

E tuttavia è anche vero che le forme di denominazione del divino nella loro polisemia, come nelle loro varianti e trasformazioni, disegnano una rete che, anche se resta difficilmente decifrabile, merita di essere ricostruita nei suoi nodi e punti di svolta e talora restituita in modo congetturale. Esse raccontano una storia che va al cuore del politeismo (o del suo «genoma» ${ }^{63}$ ), ma che ci permette di leggerne la relazione con altre forme di espressione all'interno del medesimo orizzonte culturale o sistema religioso; parlano infine di un politeismo che richiede di essere indagato non solo orizzontalmente, ma anche verticalmente, nelle sue sequenze ereditarie e nelle sue alterazioni genetiche.

\section{BIBLIOGRAFIA}

ASHERI 1988: D. Asheri, «Introduzione al libro I», in Id. (a cura di), Erodoto. Le Storie. Libro I. La Lidia e la Persia, Milano 1988, XCIX-CXIV.

ATTALI, MASSA in c.d.s., M. Attali, F. Massa, «A pagan temple, a martyr shrine, and a synagoge in Daphne: sharing sacred space in fourth century Antioch», in M. Burchardt, M. Giorda (eds), Geographies of Encounter: The Rise and Fall of Multi-Religious Spaces, Malden, MA, in c.d.s.

Belayche, BRulé ET AL. 2005: N. Belayche, P. Brulé et al. (éds), Nommer les dieux. Théonymes, épithètes, épiclèses dans l'Antiquité, Turnhout 2005.

BERGJAN, ELM 2018: S.-P. Bergjan, S. Elm (eds), Antioch II. The Many Faces of Antioch: Intellectual Exchange and Religious Diversity, CE 350-450, Tübingen 2018.

BONANNO 2013: D. Bonanno, «Coltivare e prevenire l'indignazione. Espressioni umane della nemesis nei poemi omerici», in D. Motta, N. Cusumano (a cura di), Xenia. Studi in onore di Lia Marino, Caltanissetta-Roma 2013, 13-37.

BONANNO in c.d.s.: D. Bonanno, «Némésis mystérique: L'arrière-plan philosophique de l'Hymne orphique 61 et sa réception», in F. Massa, N. Belayche (éds), Les philosophes et les mystères dans l'empire romain, Liège in c.d.s. 
BONNET 2017: C. Bonnet, «Cartographier les mondes divins à partir des épithètes. Prémisses et ambitions d'un projet de recherche européen (erc advanced grant)», Rivista di Studi Fenici 45 (2017), 49-63.

BONNET, BIANCO ET AL. 2018: C. Bonnet, M. Bianco, et al., «"Les dénominations des dieux nous offrent comme autant d'images dessinées" (Julien, Lettres 89b, 291 b). Repenser le binôme théonymeépithète», Studi e materiali di storia delle religioni 84, 2 (2018), 567-591.

BRISSON 1985: L. Brisson, «La figure de Chronos dans la Théogonie orphique et ses antecedents iraniens», in D. Tiffenau (éd.), Mythes et représentations du temps, Paris 1985 [ristamp. in: LBrisson, Orphée et l'Orphisme dans l'Antiquité gréco-romaine, Adelshot 1995], 37-55.

BRISSON 2012: L. Brisson (éd.), Platon, Phèdre, Paris 2012.

CGRN: J.-M. Carbon, S. Peels, V. Pirenne-Delforge, A Collection of Greek Ritual Norms (CGRN), Liège 2016- (http://cgrn.ulg.ac.be, consultato nel dicembre 2020).

CHIAI 2017: G.F. Chiai, Troia, la Troade ed il Nord Egeo nelle tradizioni mitiche greche, Paderborn 2017.

CHIASSON 1986: C. Chiasson, «The Herodotean Solon», Greek, Roman, and Byzantine Studies 27, 3

(1986), 249-262.

CHIASsON 2003: C. Chiasson, «Herodotus' Use of Attic Tragedy in the Lydian Logos», Classical Antiquity 22 (2003), 5-22.

CRANE 1995: G. Crane, «The Prosperity of Tyrants: Bacchylides, Herodotus, and the contest for Legitimacy», Arethusa 29, 1 (1996), 57-85.

DAUTREMER 1899: L. Dautremer, Ammien Marcellin : étude d'histoire littéraire, Lille 1899.

DeTIENNE 1977: M. Detienne, I maestri di verità nell'antica Grecia, Bari 1977, [ed. or. Paris 1967].

DILLERY 2019: J. D. Dillery, «Croesus' Great Nemesis», The Cambridge Classical Journal 65 (2019), $29-42$.

FABIANO 2019: D. Fabiano, Senza paradiso. Miti e credenze sull'aldilà greco, Bologna 2019.

FANTUZZI 2020: M. Fantuzzi, «Rhesus», A. Markantonatos (ed.) Brill's Companion to Euripides, Leiden 2020, 415-439.

FRIES 2014: A. Fries, Pseudo-Euripides, Rhesus. Edited with Introduction and Commentary, Göttingen 2014.

GAGNÉ 2013: R. Gagné, Ancestral Fault in Ancient Greece, Cambridge 2013.

GHI: R. Osborne, P.J. Rhodes (eds), Greek Historical Inscriptions 478-404 B.C., Oxford 2017.

INGARAO 2020: G. Ingarao, Scelta e necessità: la responsabilità umana in Erodoto, München 2020.

Iscr. di Cos: M. Segre, Iscrizioni di Cos, Monografie della Scuola Archeologica di Atene e delle Missioni Italiane in Oriente, 6, Roma 1993.

KRISCHER 1993: T. Krischer, «Drei Definitionen des Glücks: Pindar, Herodot, Prodikos», Rheinisches Museum für Philologie, 136 (1993), 213-222.

LEBRETON, BONNET 2019: S. Lebreton, C. Bonnet, «Mettre les polythéismes en formules ? À propos de la base de données Mapping Ancient Polytheisms», Kernos 32 (2019), 267-296.

MATTHEWs 1996: V.J. Matthews, Antimachus of Colophon, Mnemosyne Supp. 155, Leiden- New YorkKöln 1996. 
Momigliano 1974: A. Momigliano, «The lonely historian Ammianus Marcellinus», Annali della Scuola Normale Superiore di Pisa, s. III, 4, 4 (1074), 1393-1407.

MOREAU 1988: A.M. Moreau, «Étimologie et mythe originel : Adraste, Andromaque, Déjanire», in S. Geey (ed.), Sens et pouvoir de la nomination. Actes du colloque de Montpellier (23, 24 Mai 1987), Montpellier 1988, 105-124.

MosCATI - CASTELNUOVo 2016: L. Moscati-Castelnuovo, Solone e Creso. Variazioni letterarie, filosofiche e iconografiche su un tema erodoteo, Atti della giornata di studi (Macerata, 10 marzo 2015), Macerata 2016.

PARKER 1996: R. Parker, Athenian Religion. A History, Oxford 1996.

PARKER 2017: R. Parker, Greek Gods Abroad. Names, Natures, Transformations, Oakland 2017.

PAUL 2013: S. Paul, Cultes et sanctuaires de l'île de Cos, Liège, Suppl. a Kernos 28, Liège 2013.

PelLING 2006: C.B.R. Pelling, «Educating Croesus: Talking and Learning in Herodotus'Lydian Logos», Classical Antiquity 25 (2006), 141-177.

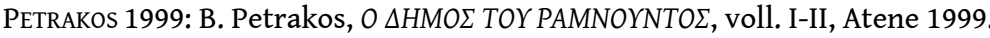

PISANo in c.d.s.: C. Pisano, «Entre qualificatifs et hétéronymes divins : les épithètes homériques à fonction théonymique», in C. Bonnet, G. Pironti (éds), Les dieux d'Homère III. Epônymiai. Questionner les dénominations divines et leur circulation, Suppl. a Kernos, in c.d.s.

POSNANSKY 1890: H. Posnansky, Nemesis und Adrasteia. Eine mythologisch-archäologische Abhandlung, Breslau 1890.

RIKE 1987: R.L. Rike, Apex omnium. Religion in the Res Gestae of Ammianus, Berkeley-Los AngelesNew York, 1987.

RoIG LANZILLOTTA 2010: L. Roig Lanzillotta, «The So-called Envy of the Gods: Revisiting a Dogma of Ancient Greek Religion», in J. Dijkstra, J. Kroesen, Y. Kuiper (eds), Myths, Martyrs and Modernity, Leiden-Boston-New York 2010, 75-93.

SCARPI 2007: P. Scarpi, Le religioni dei Misteri. Eleusi, Dionisismo, Orfismo, vol. 1, Milano 2007.

SCHEID, SVENBRo 2014: J. Scheid, J. Svenbro, La tortue et la lire. Dans l'atelier du mythe antique, Paris 2014.

SHAPIRO 1996: S.O. Shapiro, «Herodotus and Solon», Classical Antiquity 15, 2 (1996), 348-364.

SHERWIN-WHITE 1978: S.M. Sherwin-White, Ancient Cos: An Historical Study from the Dorian Settlement to the Imperial Period, Göttingen 1978.

USENER 1896: H. Usener, Götternamen. Versuch einer Lehre von der religiösen Begriffsbildung, Bonn 1896.

VAN BREMEN 2010: R. Van Bremen, «Adrastos at Aphrodisias», in R.W.V. Catling, F. Marchand, M. Sasanow, Onomatologos Studies in Greek Personal Names presented to Elaine Matthews, Oxford 2010, 441-455.

VERSNEL 2011: H.S. Versnel, Coping with the Gods. Wayward Readings in Greek Theology, Leiden-Boston 2011.

WEST 1983: M. West, The Orphic Poems, Oxford 1983. 


\section{NOTE}

1. Una prima versione di questo lavoro è stata presentata nel corso di una giornata di studi, organizzata presso l'Università Toulouse Jean Jaurès, nel giugno del 2019, in onore di Nicole Belayche, dal titolo «Stephanephoria. Honorer les dieux, couronner un parcours» e, successivamente, in un webinar, tenutosi per iniziativa del Laboratorio di Antropologia del Mondo Antico (Università di Pisa), diretto da R. Di Donato e coordinato da A. Taddei. Ai partecipanti ai due incontri desidero esprimere la mia riconoscenza per avere arricchito con le loro osservazioni la mia indagine. Ora, come allora, il mio pensiero affettuoso va a Nicole Belayche, in ricordo di un soggiorno parigino, che, grazie alla sua generosa accoglienza, ho potuto svolgere nel 2014 presso l'École Pratique des Hautes Études-Centre AnHiMA (Paris), nel quadro di un progetto finanziato dalla Fondazione A. v. Humboldt, di cui questo articolo presenta parte dei risultati.

2. Adotto la nozione di "attributo onomastico" proposta, nell'ambito dell'indagine sulle strategie di denominazione del divino, dall'équipe del progetto tolosano ERC-Mapping Ancient Polytheism. Cfr. a questo proposito, in particolare, BONNET, BIANCO ET AL. 2018, 584. Gli studi sulle modalità di denominazione del divino nel mondo antico, che, fin dal XIX sec., non hanno mancato di sollecitare l'interesse degli specialisti, si sono arricchiti negli ultimi anni di molte e importanti riflessioni: cfr., oltre all'ormai classico USENER 1896, i contributi raccolti in BELAYCHE, BRULÉ ET AL. 2005; PARKER 2017; e naturalmente i lavori che presentano i risultati raggiunti dal gruppo di Toulouse: BONNET 2017; LEBRETON, BONNET 2019.

3. Su tali questioni, si veda PISANo in c.d.s.

4. BONNET, BIANCO ET AL. 2018, 567.

5. Come affermano i colleghi dell'équipe tolosana: «Le nom n'est pas un simple label; il a une force descriptive, perceptive, cognitive et performative, en ce qu'il désigne, représente, donne à voir et à connaître, en ce qu'il fait exister et agir ce qui est nommé»: BONNET ET AL. 2018, 569.

6. È sufficiente rinviare a POSNANKY 1890.

7. Nemesis è generalmente definita Rhamnusia, con riferimento al demo dell'Attica, in cui è localizzato il suo principale luogo di culto (Call. Hymn. III 232 e Catull. 64, 395; 66, 71; 68, 77). Esiodo la designa come pema thnetoisi brotoisi nella Teoogonia (v. 223), marcandone la temibilità. Nei Canti ciprii è kallikomos (Ath. VIII 10, 32, 334c-d [= Cypria fr. 10 West]). È hyperdikos e dichoboulos in Pindaro (Ol. VIII 114 e Pyth. X 67). In epoca imperiale, la troviamo menzionata ora come ourania ora come oupis (IG I ${ }^{2} 5070$ e IGUR III 1155). Come si vedrà più avanti è spesso definita megale (cfr. infra $\S 20 ; 25 ; 28)$ o precisamente designata con attributi che fanno riferimento alla sfera semantica dell'amministrazione della giustizia e alle sue capacità sanzionatorie: cfr. rispettivamente infra la testimonianza di Platone $\S 15$ e il componimento che le è dedicato all'interno della raccolta degli Inni orfici (Hymn. Orph. 61) e dal poeta di età adrianea Mesomede (Hymn. 3). Sul profilo di Nemesis quale emerge dagli Inni orfici, mi permetto di rinviare a BONANNO in c.d.s.

8. Amm. XIV 11, 24.

9. Cfr. per esempio Schol. Eur. Rhes. 342 .

10. La figura di Adrasteia ritorna anche in un altro passo delle Res gestae (XXIII 3, 10), come colei che sorveglia le vicende umane (humanorum spectatrix). Nella raccolta degli Inni orfici (61), Nemesis è celebrata in termini molto simili a quelli che poi userà Ammiano per Adrasteia: per esempio, quale basileia («regina»); panderkes («che osserva tutto»). Ancora più marcata è la ripresa dell'Inno a Nemesis di Mesomede, la divinità è designata come figlia di Dike, capace di volgere le alterne sorti dei mortali e di abbassarne il capo che si insuperbisce (Mes. Hymn. 3, vv. 7-10: [...] ن்

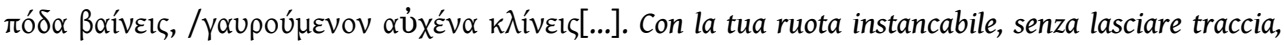
volgi la fortuna grigia degli uomini arrivi di nascosto e abbatti la testa che si insuperbisce. Trad. personale). Alcuni studiosi hanno inoltre messo in evidenza l'influenza del neoplatonismo in 
questo passo di Ammiano, soprattutto nell'uso del termine substantia corrispondente al greco hypostasis (DAUTREMER 1899, 87); altri invece hanno riscontrato nelle sue parole le linee di una posizione personale che rivela le questioni teologiche di interesse dello storico (RIKE 1987, 12-13). Momigliano 1974, 1402 sospettava che lo storico non avesse le «idee chiare» su Adrasteia.

11. Cfr. infra § 35 .

12. Il legame tra Zeus e Adrasteia è confermato anche successivamente: quest'ultima è menzionata, in relazione all'infanzia di Zeus, all'interno di uno spazio di ambientazione tipicamente cretese, come la ninfa, figlia del re Melisseo, cui Rea affidò il compito di allevare il Cronide, sottraendolo alle ire del padre Callim. H. 1, 46 e Apollod. I 1, 6. Nel III libro delle Argonautiche, Apollonio Rodio (vv. 129-141) la ricorda come colei che fece dono al fanciullo divino di un preziosissimo giocattolo, costituito da una palla metallica, ricoperta da una volta cerulea, capace di tracciare, se lanciata in aria, una scia luminescente come una stella, quasi fosse una metafora del cosmo, che egli un giorno sarebbe stato chiamato a governare.

13. Cfr. DETIENNE 1977.

14. La prima volta, il nome di Adrasteia ricorre all'interno di una dichiarazione del Coro che la invoca, proclamando contestualmente la sua fiducia nell'intervento risolutore di Reso, atteso come un liberatore e paragonato allo stesso Zeus (Eur. Rhes. 342); la seconda volta, è il sovrano tracio stesso, a chiamarla in causa, prima di illustrare il progetto eccessivamente ambizioso di portare guerra agli Argivi e alla Grecia intera, una volta liberata Troia dall'assedio acheo (ibid. v. 468). Sul Reso pseudoeuripideo con una proposta interessante di datazione al IV sec. cfr. FANTUZZI 2020; sui versi in particolare in cui si evoca il nome di Adrasteia, si veda il recente commento all'opera: FRIES 2014 ad loc.

15. Cfr. IG I $\mathrm{I}^{3} 369,1.67$ e 383, l. 67; GHI 160. Cfr. su queste iscrizioni cfr. le considerazioni di PARKER 1996, 172 e 195.

16. Paus. I 33. Su Ramnunte e sul santuario di Nemesis, PETRAKos 1999.

17. Plat. Phaedr. $248 \mathrm{c}-\mathrm{d}=$ fr. 20, 1 Bernabé. Nella raccolta di SCARPI 2007, 427, intitolata Le religioni dei misteri, il passo è inserito all'interno della rubrica "Escatologia e soteriologia" e l'Adrasteia del Fedro platonico definita nel commento «il fondamento mitico della teoria della reincarnazione» (p. 668). WEST 1983, 195-196, 54 n. 10 e BRISSON 1985, 54 n.10 che sottolineano come l'Adrasteia del Fedro svolga lo stesso ruolo di Lachesis nel mito di Er all'interno della Repubblica (Plat. Rp. X 620d) e sostengono l'equivalenza di Nemesis e Adrasteia nel V sec.

18. Plat. Phaedr. 261a.

19. Sul Fedro di Platone, cfr. l'introduzione di BRISSON 2012.

20. Plat. Phaedr. 248b-249c.

21. Platone stigmatizza, senza mezzi termini, il sofista, come un «atleta dei discorsi» e esperto dell'arte della contraddizione e come un illusionista capace di ingannare i giovani con i suoi discorsi (Plat. Soph. $231 \mathrm{~d}$-e; $234 \mathrm{c}$-e). Nel Gorgia invece la sofistica è considerata una forma di adulazione (Plat. Gorg. 463 b). Abile con la parola dovrà essere necessariamente anche il tiranno, descritto come incline, nei suoi primi giorni di governo, a mostrarsi amabile e sorridente e facile alle promesse (Plat. Rp. VIII 566d-e).

22. Plat. Rp. V 451a.

23. L'Inno a Demetra di Callimaco narra, per esempio, la vicenda del figlio del sovrano tessalo Triopa, Erisittone che aveva tagliato il bosco sacro alla dea per costruire un palazzo in cui organizzare dei banchetti. Rimproverato dalla dea, che aveva preso le sembianze della sacerdotessa Nicippe, reagì minacciandola con la sua ascia e rivolgendole parole empie che

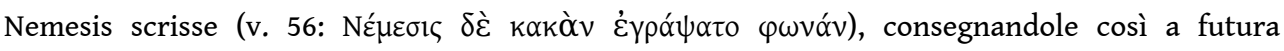
memoria. La punizione implacabile di Demetra condannò il giovane a una fame insaziabile.

24. Plat. Leg. $717 \mathrm{c}-\mathrm{d}$. 
25. L'episodio ha sollecitato tante riflessioni da parte degli studiosi e degli intellettuali di diverse epoche. Sulla fortuna del dialogo, si vedano i contributi raccolti nel volume a cura di MoscATI Castelnuovo, 2016. Sulla figura di Creso nelle Storie, cfr. PelLing 2006.

26. Hdt. I $30,2$.

27. Il primo esempio evocato da Solone è rappresentato dall'Ateniese Tello che, nel corso della sua vita, aveva vissuto in una città florida e, partecipando gloriosamente alla sua difesa, aveva terminato la sua esistenza circondato da una progenie fortunata e tra gli onori dei suoi concittadini (Hdt. I 30,4). Il secondo posto, nella graduatoria della felicità, il legislatore lo assegna ai giovani argivi Cleobi e Bitone, esempio di devozione e amore filiale, che con le loro forze trasportarono su un carro la madre per 45 stadi, fino al tempio di Era, consentendole così di prendere parte alla festa in onore della dea. I due, una volta giunti al santuario tra l'ammirazione dei presenti e della madre, dopo aver sacrificato e banchettato si addormentarono nel tempio, senza svegliarsi più. Gli Argivi eressero in loro onore due statue che dedicarono a Delfi (Hdt. I 31).

28. Hdt. I 32.

29. Hdt. I 33.

30. I termini della distanza che separa Solone e Creso nella concezione della felicità sono stati più volte analizzati: cfr. con particolare riferimento al lessico impiegato da Erodoto, CHIASSON 1986; KRISCHER 1993, 218-219 e CRANE 1996.

31. Hdt. I 216, 3; II 6, 2; II 40, 3; II 95,1; II 121, $1 \alpha$; III 66, 1; IV 194; V 23, 2; VI 132; VII 83, 2; VII 190, 1; IX 51,4. Per un'analisi lessicale dell'uso di phthonos in Erodoto con una sintesi del dibattito sul tema, INGARAO 2020, 130.

32. Hdt. VII 10, 3 e $10 \zeta$.

33. Hdt. VII 10, e in part. $\varepsilon$ (trad. personale).

34. Per un'interpretazione dello phthonos theon e una storia degli studi su questa categoria che attraversa gran parte della letteratura greca, si leggano le riflessioni di RoIG LANZILLOTTA 2010.

35. Per un tentativo di schematizzazione delle forze che incidono sul corso dell'esistenza umana, nell'ambito delle Storie erodotee, VERSNEL 2011, 179-187. Sulla complessità dei regimi di causalità in opera nelle Storie erodotee, cfr. da ultimo INGARAO 2020 con ampia e approfondita discussione della bibliografia.

36. Hdt. I 13 e 91.

37. Il carattere programmatico del primo libro delle Storie di Erodoto è stato più volte sottolineato: ASHERI 1988, XCIX e CI sottolinea come esso costituisca, in qualche modo, la «quintessenza» di tutta l'opera e, in particolare, a proposito del dialogo tra Solone e Creso precisa come esso serva a delineare il profilo tragico di Creso. Sull'importanza del discorso di Solone a Creso insiste SHAPIRO 1996, 362 che rileva come esso illustri sostanzialmente i caratteri della relazione tra divino e umano e come possa costituire il quadro filosofico di tutta l'opera erodotea. Sulla vicenda di Creso e sui suoi rapporti con la tragedia attica, CHIASson 2003.

38. Hdt. I 34-45. A lungo l'Adrasto erodoteo, il cui nome ricorre nell'opera nella forma ionica

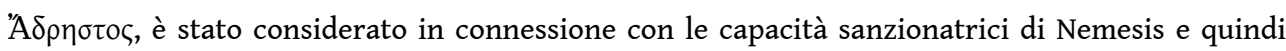
individuato quale trait d'union con Adrasteia. Analogie sono state ravvisate tra la storia del giovane Atys figlio di Creso, ucciso nel corso di una caccia al cinghiale, dalla punta di un giavellotto scoccato proprio dal frigio Adrasto, ospite del padre che aveva promesso di proteggerlo e il mito di Cibele ed Attis, quest'ultimo, morto anch'esso, in Lidia, per opera di un cinghiale inviato da Zeus (Paus. VII 17, 9-10). In realtà, a parte queste somiglianze superficiali, mancano riscontri concreti nelle fonti. Per un'interpretazione, in tal senso, si può vedere anche recentemente DILLERY 2019.

39. A questo proposito, mi permetto di rinviare nuovamente a un mio lavoro precedente: BONANNO 2013, 33-34.

40. Hdt. I $32,1$. 
41. Hdt. I 86.

42. Cfr. infra, Harpokr. s.v. Adrasteia=Antimach. fr. 131 Matthews fr. 53 Wyss.

43. A proposito di questa regione della Troade, cfr. CHIAI 2017, 108-110.

44. Hom. Il. II 825-834.

45. Hom. Il. II 834 (trad. R. Calzecchi Onesti - Einaudi).

46. MATthEWS 1996, 314-321.

47. Apollod. III 12,3.

48. Paus. Att. s.v. Adrasteia; Zen. I 30; Ael. Dion. s.v. Nemesis Adrasteia; Diogen. s.v. Adrasteia Nemesis; Suid. s.v. Adrasteia Nemesis.

49. Hdt. IV 142 e Dion. Chrys. Or. 37, 10.

50. MoReAu 1988, 108-113 e VAN BREMEN 2010, 450.

51. Cfr. Chrys. SVF II, 169 fr. 528 e Plut. De sera num. vindicta 25, 564e in cui Adrasteia è designata come la figlia di Zeus e Ananke. Un'analoga visione è esposta anche nel trattato pseudoaristotelico De mundo, VII 401b, 8-14.

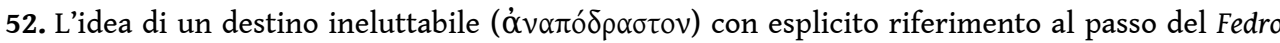
platonico la si ritrova nel trattato, di dubbia paternità plutarchea, De fato, 568 b-c. Negli Stromati dell'autore cristiano Clemente Alessandrino, Adrasteia assicura efficacia ai comandamenti divini, rendendo impossibile sottrarsi a dio (Clem. Alex. Strom. III 20, 8:

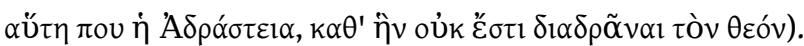

53. Nella città di Antiochia nel sobborgo di Daphne, secondo Giovanni Malala, all'interno dello stadio, vi era un santuario di Nemesis fatto costruire da Diocleziano: Ion. Malal. 307. Sulla città di Antiochia, in età tardoantica, si vedano i contributi recentemente raccolti in BERGJAN, ELM 2018. Su Antiochia e il sobborgo di Daphne come luogo di interazione e competizione tra diversi culti nel IV sec. d.C., cfr. ATTALI, MASSA in c.d.s.

54. Apollod. III 7, 2-4.

55. A tal proposito è sufficiente fare riferimento ai temi affrontati nel De sera numinis vindicta di Plutarco. Sul contributo dell'opera di Plutarco all'organizzazione delle tradizioni greche relative al tema della giustizia divina, cfr. GAGNÉ 2013, 33-44. Sulle modalità di attuazione, fondamentalmente terrene, di tale giustizia, si vedano invece le considerazioni di FABIANO 2019, 127-165.

56. La menzione congiunta delle due divinità è attestata epigraficamente anche altrove, ma purtroppo il carattere isolato di queste testimonianze e, talora, la difficoltà di trarre informazioni

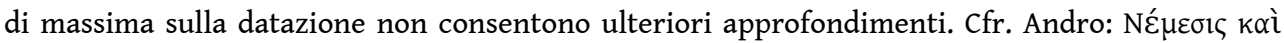

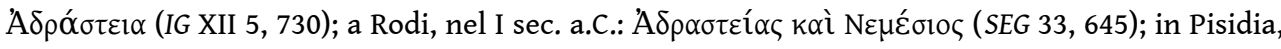

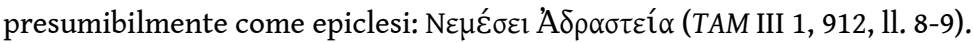

57. Entrambe le iscrizioni sono state messe in relazione alla vendita di sacerdozi: effettivamente IG XII 4, 325 presenta sulla faccia posteriore il contratto per la vendita di un sacerdozio, senza la menzione delle divinità che tuttavia gli studiosi danno per scontato debba riferirsi a Nemesis e Adrasteia: cfr. il commento all'iscrizione in CGRN 142; IG XII 4, 318 è invece estremamente frammentaria e la menzione della vendita del sacerdozio è frutto di corpose integrazioni: cfr. Iscr. di Cos, ED 144.

58. Diversa l'opinione di PAUL 2013, 153-156 che ritiene che l'indicazione dei banchieri nel gruppo dei sacrificanti e l'ammontare eccessivamente elevato dell'ammenda da pagare alle divinità possa essere interpretato come un indizio di una competenza di Adrasteia e Nemesis nella sfera della retribuzione di tipo pecuniario. L'ipotesi è suggestiva, ma non tiene conto del fatto che l'indicazione della sanzione è messa in relazione con eventuali trasgressioni da parte di affrancati e impresari e come conseguenza di una colpa precisa. SHERWIN-WHITE 1978, 325 ritiene invece che Adrasteia e Nemesis presiedessero a Cos le manomissioni degli schiavi, in ragione del loro rapporto con il destino umano. 
59. A proposito dei nomi parlanti SCHEID, SVENBRo 2014, 24, affermano: «On peut même dire que ce sont précisément la polysémie et l'ambiguïté syntaxique qui font leur force, dans la mesure où elles permettent la mise en œuvre d'exégèses successives». Sulla funzione del nome proprio come «generatore di miti»: cfr. anche ibid. 159 e ss.

60. Men. Sam. 501-505; Alciphron, IV 6, 5; Luc. Ap. VI 10.

61. Il significato di tale rituale meriterebbe una riflessione più approfondita di quello che lo spazio limitato di una nota possa consentire e mi riprometto di tornare sull'argomento in un'altra occasione. Tuttavia, il confronto con altre fonti può servire a decodificare meglio le istanze sottese a questa prassi. L'aspetto catartico dell'atto di sputare emerge dal passo di Apollonio Rodio IV 477-480, in cui Giasone, dopo l'uccisione a tradimento di Apsirto, lecca tre volte la spada, sputando fuori dai denti, tre volte, la maledizione, come è consueto per rendere propizio un omicidio a tradimento per coloro che coloro che lo hanno commesso: [...]

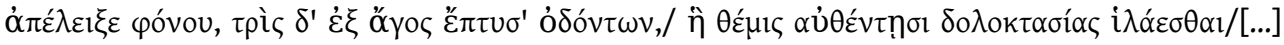
(«[...] e per tre volte sputò il sangue, sputando la sozzura, comesi conviene a chi deve espiare un'uccisione compiuta/a tradimento [...], trad. A. Borgogno-Mondadori»). Lo sputo come gesto di ostilità lo ritroviamo nell'Antigone di Sofocle, quando Creonte suggerisce al figlio Emone di lasciare la giovane donna, sputandole addosso, come una nemica (v. 653), ma sarà poi proprio lui, davanti al cadavere di Antigone, morta per impiccagione, a sputare in faccia al padre e sguainare la spada, con l'intento di ucciderlo (v. 1232). Cfr. con un analogo significato, anche Diog. Laert. VI 32 e Plut. Reg. et imper. apophth. 189 a. Plinio (Nat. hist. XI 251) riporta invece un'altra pratica rituale connessa a Nemesis, quella di portare l'anulare sotto l'orecchio destro, sede della memoria, dopo averlo portato alla bocca, per nascondervi un pensiero di cui si chiede perdono agli dèi.

62. Greg. Paroem. s.v. eis to kolpon ptuein.

63. BONNET, BIANCO ET AL. 2018, 568.

\section{RIASSUNTI}

Prendendo le mosse da un passo di Ammiano Marcellino (XIV 11,25), che lascia emergere un'evidente sovrapposizione tra la figura di Adrasteia e quella Nemesis, il contributo si propone di esaminare le ragioni che hanno condotto le due potenze ad essere indicate l'una come l'eteronimo dell'altra. A partire dalle fonti di V secolo fino alla paremiografia e alla lessicografia di età imperiale si ricostruisce un percorso che si propone di mostrare gli accidenti e le molteplici variabili cui le logiche di denominazione del divino, nel mondo greco, possono essere soggette.

Starting from a passage by Ammianus Marcellinus (XIV 11,25), which reveals an overlapping between Adrasteia and Nemesis, the contribution aims at examining the reasons that led the two goddess to be indicated one as the heteronym of the other. Taking into considerations the sources from fifth-century up to paremiography and lexicography of the imperial age, a path is reconstructed that shows the many accidents and the variables to which the naming of the divine may be submitted, in the Greek world. 
INDICE

Keywords : Nemesis, Adrasteia, Herodotus, Croesus, Adrastos

Parole chiave : Nemesis, Adrasteia, Erodoto, Creso, Adrasto

\section{AUTORE}

\section{DANIELA BONANNO}

Università degli Studi di Palermo

Dipartimento Culture e Società

Viale delle Scienze, Ed. 15

90128

Palermo

daniela.bonanno(at)unipa.it 